\title{
Experimental investigations of turbulent drag reduction by surface-embedded grooves
}

\author{
B. FROHNAPFEL, J. JOVANOVIĆ AND A. DELGADO \\ Institute of Fluid Mechanics, Friedrich-Alexander University of Erlangen-Nürnberg, Cauerstrasse 4, \\ 91058 Erlangen, Germany
}

(Received 24 April 2007 and in revised form 6 August 2007)

Consideration of near-wall turbulence in the functional space that emphasizes the level of anisotropy of the velocity fluctuations not only provides an understanding of the causative physics behind remarkable effects of turbulent drag reduction, but also leads to the logical design of a surface topology which is shown experimentally to be capable of producing a significant reduction of viscous drag which far exceeds what has been achieved so far.

\section{Introduction}

A significant portion of the drag which counteracts the motion of a body through a fluid is generated in the thin, viscous region close to the solid boundary where the flow is nearly always turbulent. The viscous contribution to the total drag amounts to about $50 \%$ on commercial aircraft, $90 \%$ on underwater vehicles and almost $100 \%$ for pipe flows (Lee et al. 2001). If we consider current trends in the expansion of public transport worldwide and initiatives for reduced pollution of the environment, the benefs of reducing viscous drag and therefore fuel consumption are obvious.

The question of how wall-bounded turbulent flows can be rationally controlled with reasonable cost in order to reduce viscous drag has been investigated by employing different techniques such as polymer and surfactant additions, riblets, largeeddy breakup devices and compliant surfaces. More recently, futuristic techniques based on active control of near-wall turbulence employing MEMS devices equipped with sensors and actuators that are embedded in the wall have been explored, in particular with the addition of direct numerical simulations that have emerged as powerful tool in contemporary turbulence research (Bewley, Moin \& Temam 2001). Although substantial work has been done along these lines, only marginal success has been achieved for wider engineering applications: according to Walsh (1980), surface-mounted longitudinal riblets which are implementable in practice provide drag reduction of $8 \%$.

In this paper, a novel technique for passive flow control of near-wall turbulence is proposed and tested experimentally, which is based on modifications of the surface topology in an attempt to achieve drag reduction that is substantially higher than has been obtained with riblets so far. This is accomplished by forcing near-wall turbulence to satisfy axisymmetry at large and small scales very close to the wall with invariance under rotation about the axis aligned with the mean flow. Theoretical work by Jovanović \& Hillerbrand (2005) and Jovanović et al. (2006) shows that kinematic constraints imposed by local axisymmetry force turbulence very close to the wall to 


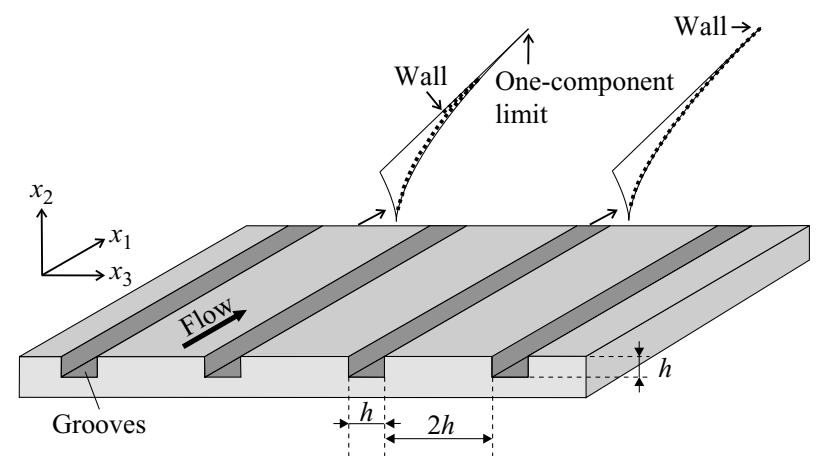

FIGURE 1. Grooves in the wall that are aligned in the flow direction force turbulence in the grooves towards the one-component limit. Sketches show expected trajectories across anisotropy-invariant maps of Lumley \& Newman (1977) for each portion of the surface.

tend towards the one-component state, and when turbulence reaches this limiting state it must be entirely suppressed across the viscous sublayer. For this special state of wall turbulence, it was shown that the turbulent dissipation rate vanishes at the wall, forcing the average energy dissipation, which balances the work done against the wall shear stress per unit mass of fluid, to reach the minimum value and then to induce a large drag reduction effect. These deductions are well supported by all available data from direct numerical simulations and numerical experiments carried out with boundary conditions imposed in order to satisfy axisymmetry close to the wall as demonstrated by Frohnapfel et al. (2007).

Based on the results discussed above, the grooves shown in figure 1 are suggested as a surface modification in order to obtain high drag reduction. Inside a groove, the velocity fluctuations in both the normal and tangential direction are suppressed due to the sidewalls and therefore it is expected that turbulence in the groove will tend towards an axisymmetric state and reach the one-component limit at the wall, which is required in order to minimize the energy dissipation and then induce the drag reduction effect. In this way the artificial boundary conditions that were shown to produce high drag reduction in numerical experiments (Frohnapfel et al. 2007) are realized locally on the channel walls. The experiment thus demonstrates how the previous analytical and numerical investigations can be successfully implemented in practical applications.

\section{Experimental set-up and measuring techniques}

The set-up for experimental investigations of turbulent drag reduction is sketched in figure 2. The flow was driven by a centrifugal blower, which was controlled by a frequency converter so that the rotation rate of the fan could be adjusted. The air stream was guided through a well-designed settling chamber consisting of alternate perforated plates and honeycombs to ensure uniform flow conditions at the outlet, which was smoothly connected to the channel. On the suction side of the blower, a Venturi nozzle allowed the determination of the volume flow rate according to Bernoulli's equation. The channel was $L=3.5 \mathrm{~m}$ long and $B=300 \mathrm{~mm}$ wide and could be adjusted to heights of $H=25$ and $35 \mathrm{~mm}$. At the channel inlet a trip was installed to ensure turbulent flow conditions and reproducible results at low Reynolds number. A trip of $12 \%$ blockage ratio was used for all drag reduction experiments by inserting thin metal sheets that protruded from the channel top and bottom walls. 


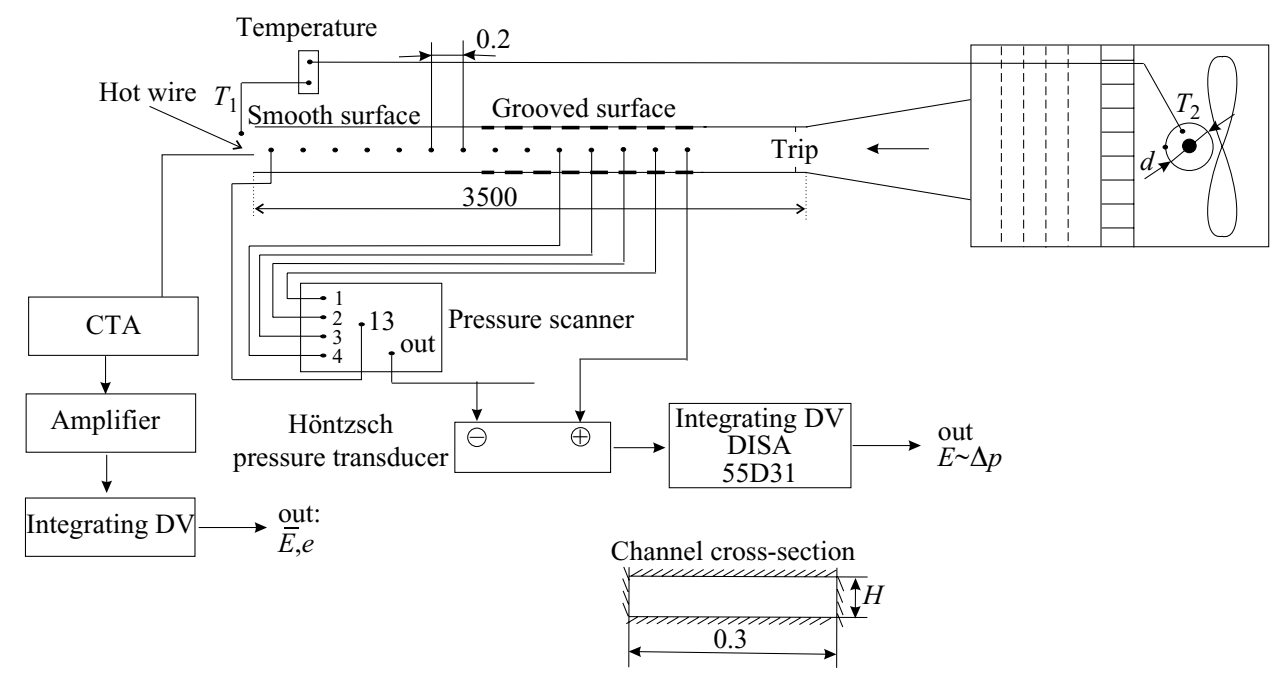

FIGURE 2. Experimental set-up.

This blockage ratio was close to $15 \%$, which was suggested by Durst et al. (1998) for turbulent properties to be independent of upstream flow conditions. After the trip, there was a length of $0.7 \mathrm{~m}$ for the flow development, which corresponds to $x_{1} / H=28.0$ and 20.0 .

The channel test section was equipped with 14 pressure taps of $300 \mu \mathrm{m}$ diameter at intervals of $200 \mathrm{~mm}$ on both of the channel sidewalls. Pressure tubes were connected to each pressure tap and were guided over the pressure scanner to the calibrated Höntzsch transducer, which had a total measuring range of $100 \mathrm{mmH}_{2} \mathrm{O}$. Using different gain settings, it could be adjusted to higher resolutions resulting in measuring ranges of $30,10,3$ and $1 \mathrm{mmH}_{2} \mathrm{O}$. For measurements of ultra-low pressure differences, the signal from the transducer was additionally amplified between 10 and 50 times in order to increase the resolution of measurements.

For the determination of the pressure drop over the channel length, the static pressure at the first measuring station (i.e. closest to the inlet of the channel) was compared with readings from subsequent measuring stations. The test section was divided into two parts of equal length. In each part, the top and bottom walls are interchangeable so that smooth and grooved channel walls can be installed. For drag reduction measurements, the pressure drop in the portion of a channel with grooved walls was compared with that to smooth walls under identical flow conditions. This in-line set-up yields operating conditions with a high degree of similarity that cannot be achieved by running two consecutive experiments with different wall structures. The comparison of the pressure drops $\Delta p / \Delta l$ over the smooth and the grooved surface parts can be converted into drag reduction by

$$
D R=1-\frac{(\Delta p / \Delta l)_{\text {grooved }}}{(\Delta p / \Delta l)_{\text {smooth }}},
$$

which is a conservative estimate since the influence of the smooth sidewalls in the grooved channel part was not taken into account.

The bulk flow velocity $\bar{U}_{b}$ in the channel was determined from the pressure difference $\Delta p_{0}$ between the static pressure at the inlet of the blower and the ambient 


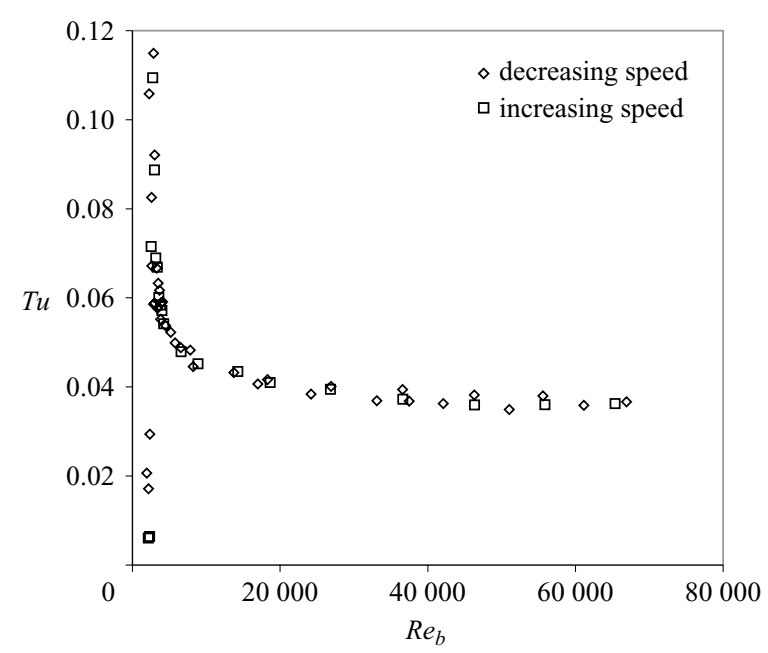

FiguRE 3. Turbulence intensity as a function of Reynolds number measured for different channel heights and for increasing and decreasing Reynolds number.

pressure according to

$$
\bar{U}_{b}=\sqrt{\frac{2 \Delta p_{0}}{\rho}} \frac{A_{\text {nozzle }}}{A_{\text {channel }}},
$$

where $\rho$ is the air density at the given temperature and atmospheric pressure and $A_{n o z z l e}$ and $A_{\text {channel }}$ are the cross-sectional areas of the nozzle inlet and the channel test section, respectively. Insertion of grooves in the second part of the test section increases the channel cross-sectional area by less than $0.5 \%$ so that the bulk velocity throughout the test section is nearly constant for a constant temperature flow. The temperature was monitored at the nozzle inlet and the channel exit and differed only by fractions of a degree. The bulk Reynolds number $R e_{b}$ of the channel flow is based on the bulk velocity $\bar{U}_{b}$, the channel height $H$ and the air viscosity $v$ at the given temperature:

$$
R e_{b}=\frac{\bar{U}_{b} H}{v} .
$$

A hot wire operated in constant-temperature mode was placed at the outlet of the test section to measure the turbulence intensity in the centre of the channel as a function of Reynolds number. Based on these measurements, the transition Reynolds number from laminar to turbulent flow was determined. Since a laminar flow has significantly lower viscous drag than a turbulent flow, as the surface structure is designed to yield drag reduction in turbulent flows, it is important to know whether measurements are taken in the laminar or turbulent flow regime. Figure 3 shows the results obtained for increasing and decreasing flow rates and different channel heights. All results fall on one curve, which suggests that transition from laminar to turbulent (and vice versa) is not influenced by either the channel height or a hysteresis effect, i.e. a difference in the transition Reynolds number for increasing and decreasing flow rates.

For high Reynolds numbers, the turbulence intensity is of order order $T u=4 \%$. Below $R_{b} \approx 10000$, the turbulence intensity increases and reaches a maximum around $R e_{b} \approx 2300$, in agreement with the experimental results of Fischer (1999). It is possible to explain these high turbulence intensities by intermittency between laminar and 

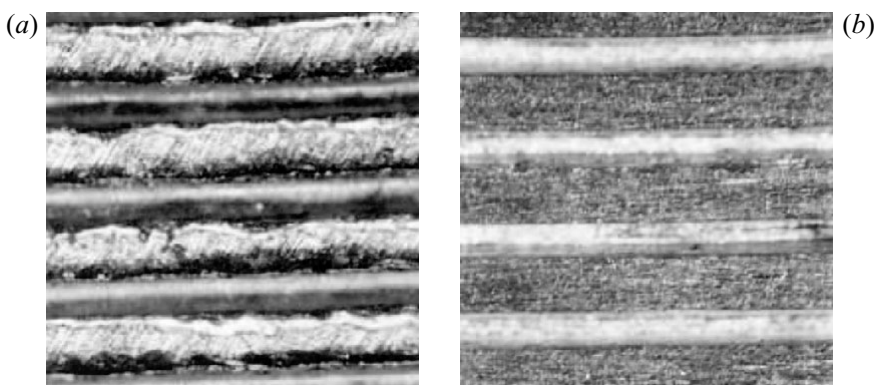

FIgURE 4. Surface patterns of the grooved channel. (a) The original surface pattern. $(b)$ The surface pattern after polishing.

turbulent flow states, since a laminar flow with a parabolic velocity profile has a higher centreline velocity than a turbulent flow at the same Reynolds number. To ensure turbulent flow conditions, the Reynolds number must be above that for which the highest turbulence intensities are measured. Thus $R e_{b}$ has to exceed $R e_{b} \approx 2300$ for the flow to be considered fully turbulent.

\section{Grooved surface}

Preliminary numerical simulations (Frohnapfel 2007), showed that grooves with square cross-section were most promising. Spacing between the grooves is designed to avoid local peaks of high wall shear stresses close to edges of the grooves (Choi, Moin \& Kim 1993). However, increased spacing reduces the theoretically possible maximum drag reduction, which can be estimated by assuming that the wall shear stress inside the groove is negligible in comparison with that acting along the flat portion of the surface. For the experiments grooves of square cross-section were used that are separated by twice their width. For this surface topology, the theoretical maximum drag reduction is $(D R)_{\max }=33 \%$.

Theoretical considerations, elaborated by Frohnapfel (2007), showed that a significant reduction in skin friction drag will occur only for infinitesimally small grooves which cannot produce secondary flow. The dimensions of such grooves are expected to scale with the wall variables, based on the wall shear velocity $u_{\tau}$ and the kinematic viscosity of the flow medium $v$, and to be of the order of the viscous length scale $h \sim v / u_{\tau}$. In an initial attempt to study the drag reduction effect, such grooves were produced by conventional milling technology which restricted minimum groove dimensions to $h \approx 150 \mu \mathrm{m}$, with a separation of $2 h \approx 300 \mu \mathrm{m}$.

The grooved surface pattern was produced mechanically on flat plates of dimensions $1.5 \mathrm{~m} \times 0.3 \mathrm{~m}$, used as top and bottom walls in the front part of the channel test section. The mechanical milling resulted in a rough, irregular surface structure, as can be seen on figure $4(a)$. The surface was then polished, resulting in the structure shown on figure $4(b)$. This final surface structure had a geometry with slightly trapezoidal grooves, as sketched in figure 5 .

\section{Experimental results}

Preliminary experiments were carried out within the channel test section with smooth walls to determine the linearity of the measurements. In figure 6 , the pressure drop over the entire test section at $R e_{b}=2850$ and 15800 is plotted. The slope of 


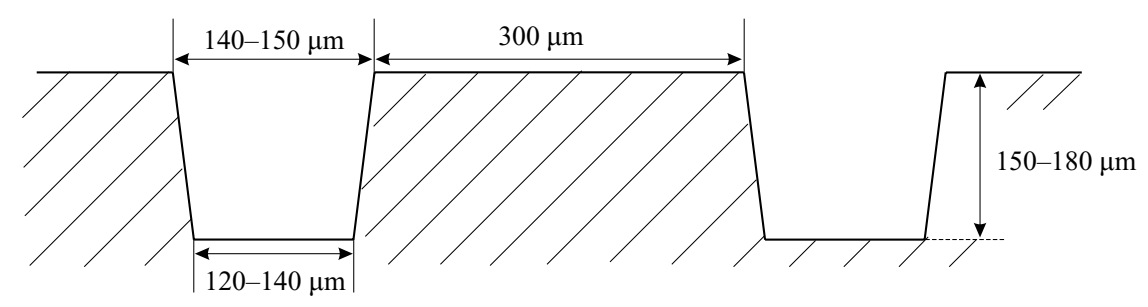

FIGURE 5. Dimensions of the polished surface with grooves.
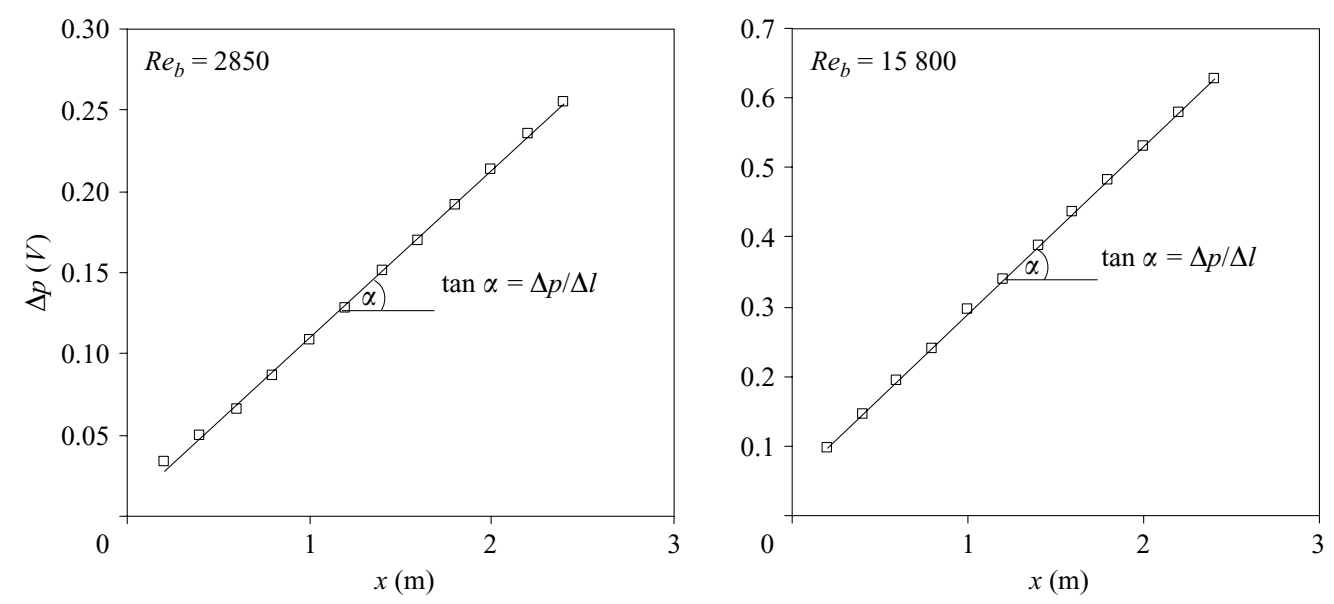

Figure 6. Pressure drop measurements over the entire test section with smooth walls at different Reynolds numbers.

the best-fit curve through all data points is used to determine the pressure gradient $\Delta p / \Delta l$. Crucial factors in the accuracy of the measurements are the linearity in the pressure drop and therefore the correct determination of the pressure gradient. The linearity decreases for low speeds where a very low pressure drop over the entire test section exists. If drag reduction is calculated for the set-up with smooth plates in both channel parts in the same way as for that with grooved walls, values up to $\pm 3 \%$ are determined for low Reynolds numbers, demonstrating the limitation of the measuring accuracy.

For the drag reduction measurements, one part of the channel needs to be equipped with grooved walls. To determine which part, the two alternative alignments of smooth and grooved parts were tested. The measurement results show in figure 7 that the set-up in which the first part of the test section has grooved walls top and bottom (and the second part has smooth walls top and bottom) yielded slightly lower values of drag reduction than the reverse configuration. It was decided to run the experiments with this set-up as it resulted in a more conservative estimate of drag reduction.

The drag reduction measurements were carried out for channel heights $H=25$ and $35 \mathrm{~mm}$. Typical measurement results are shown in figure 8 . All correspond to turbulent flow conditions. The best-fit lines closely follow the trend in the pressure drop for the grooved channel part (solid line) and the flat channel part (dashed line). Both lines are extended over the entire length of the channel for better comparison. The slope of each line represents the pressure drop $\Delta p / \Delta l$ in the corresponding part of the test section. The ratio of these two slopes is used to calculate the drag reduction 


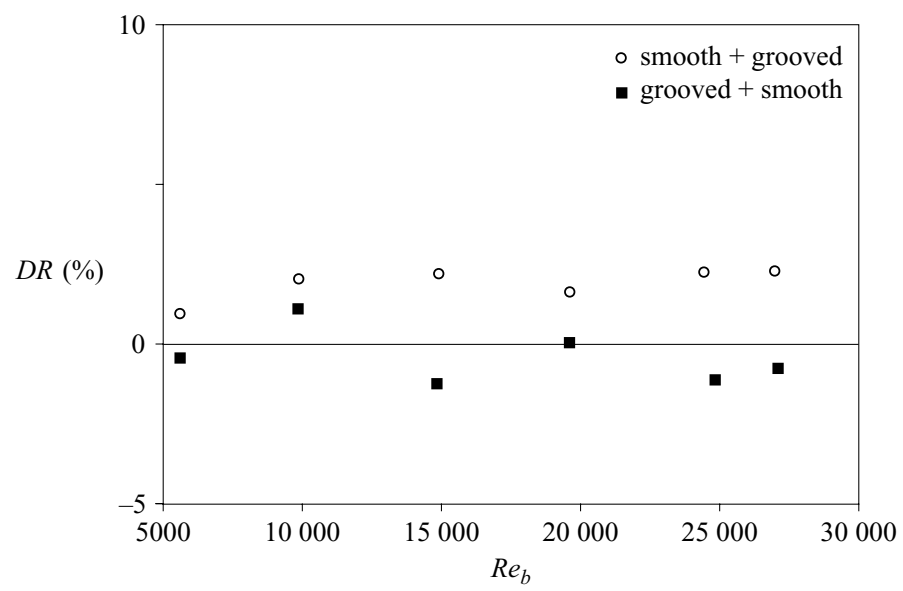

FIGURE 7. Drag reduction measurements in the channel test section with the two alternative alignments of smooth and grooved channel walls.
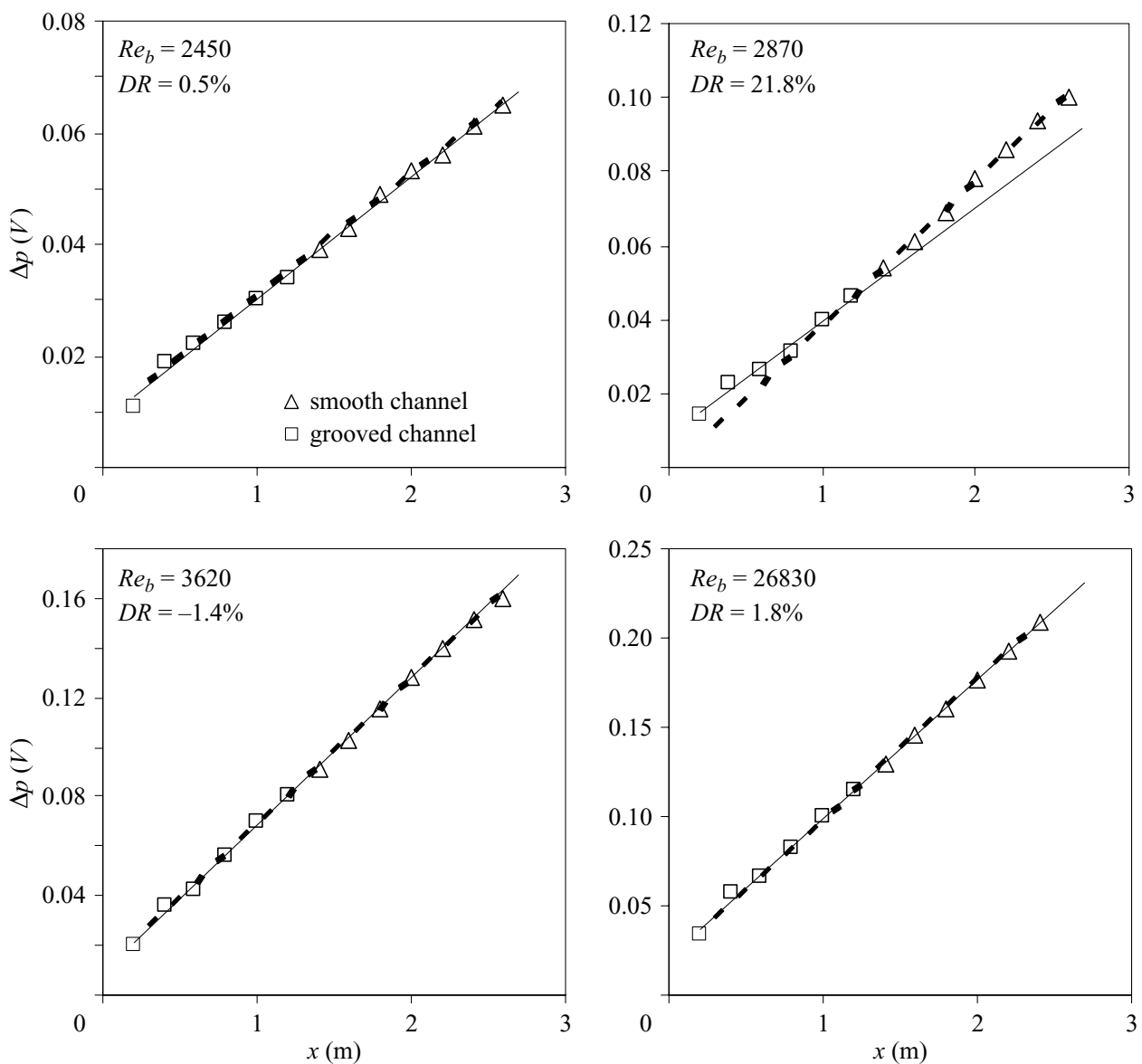

FIGURE 8. Pressure drop measurements at different Reynolds numbers over the entire test section where the first half has grooves and the second half smooth walls. For each part of the test section the pressure gradient is determined by a best-fit line through the measured data. The comparison of the corresponding slopes is used to calculate the drag reduction. 

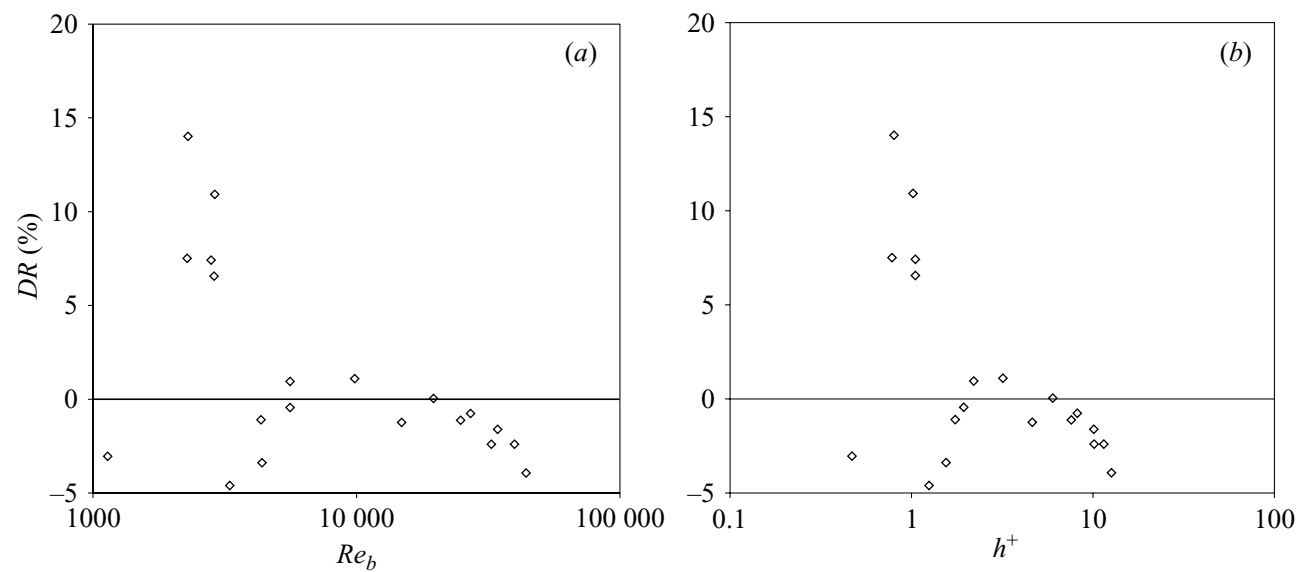

Figure 9. Drag reduction obtained with a channel height $H=25 \mathrm{~mm}$ plotted versus (a) Reynolds number and $(b)$ dimensionless size of the grooves.

as given by equation (2.1). Figure 8 demonstrates nicely that significant changes in pressure drop between the two channel sections were only found in a narrow Reynolds number range whereas the measurements at lower and higher Reynolds numbers yielded almost constant pressure gradients along the entire channel.

For the channel height $H=25 \mathrm{~mm}$, figure $9(a)$ shows all drag reduction data obtained for a wide Reynolds number range of $R e_{b} \approx 1000-45000$. In the range $R e_{b} \approx 5000-45000$, the measured drag reduction deduced from pressure drop measurements was nearly within the limit of the measurement accuracy. However, at low Reynolds numbers, $R e_{b}=2300-2900$, high drag reduction up to $14 \%$ was obtained based on repeatable pressure drop measurements. Since high drag reduction was measured for flow conditions very close to those for transition from the laminar to turbulent state to occur, fluctuations at the channel outlet were monitored during these measurements and it was found that the flow was indeed turbulent, showing no intermittency effects in the hot-wire signal.

For very low Reynolds numbers where the flow was laminar, a slight increase in drag was measured. This result is not surprising since the grooves were designed to alter the turbulent structure in the near-wall region. For a laminar flow state, the only effect of the structured surface is expected to be caused by an increase in surface area which directly results in a higher overall shear force and thus increased pressure drop.

Figure $9(b)$ shows drag reduction data for $H=25 \mathrm{~mm}$ scaled in terms of the dimensionless groove size $h^{+}$obtained according to

$$
h^{+}=\frac{h u_{\tau}}{v},
$$

where $u_{\tau}$ was calculated from pressure drop measurements in the smooth part of the channel (width $B$, height $H$ ):

$$
u_{\tau}=\sqrt{(\Delta p / \Delta l)_{\text {smooth }} \frac{B H}{2 \rho(B+H)}} .
$$

It can be seen that high drag reduction was obtained in a very narrow Reynolds number range only, in which the dimensionless size of the grooves was slightly smaller than $h^{+}=1$. 

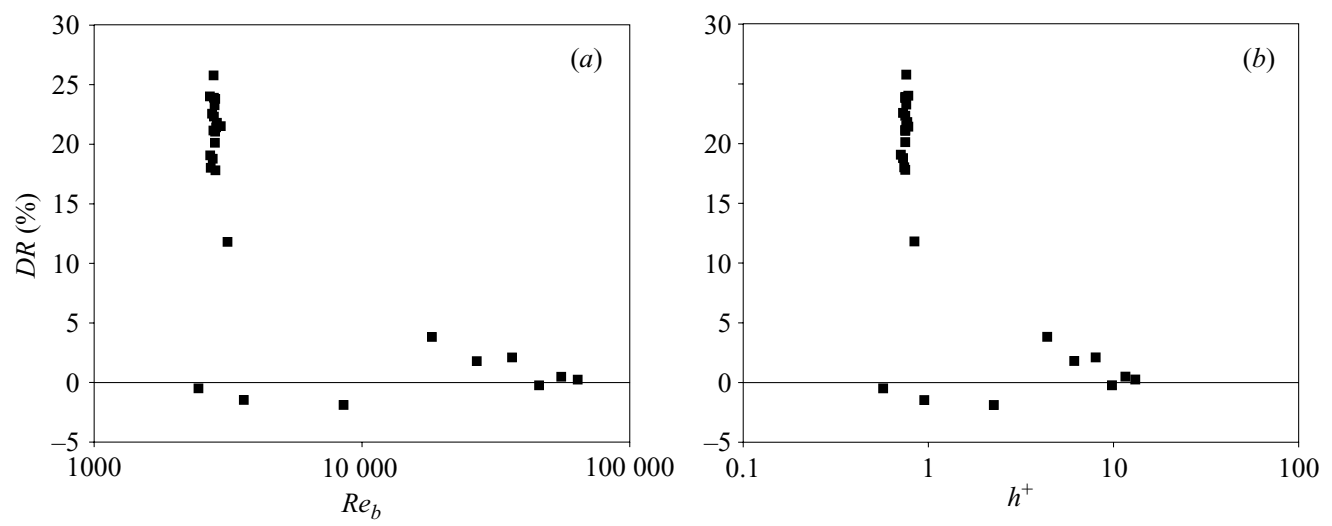

FIGURE 10. Drag reduction obtained with a channel height $H=35 \mathrm{~mm}$ plotted versus $(a)$ Reynolds number and $(b)$ dimensionless size of the grooves.

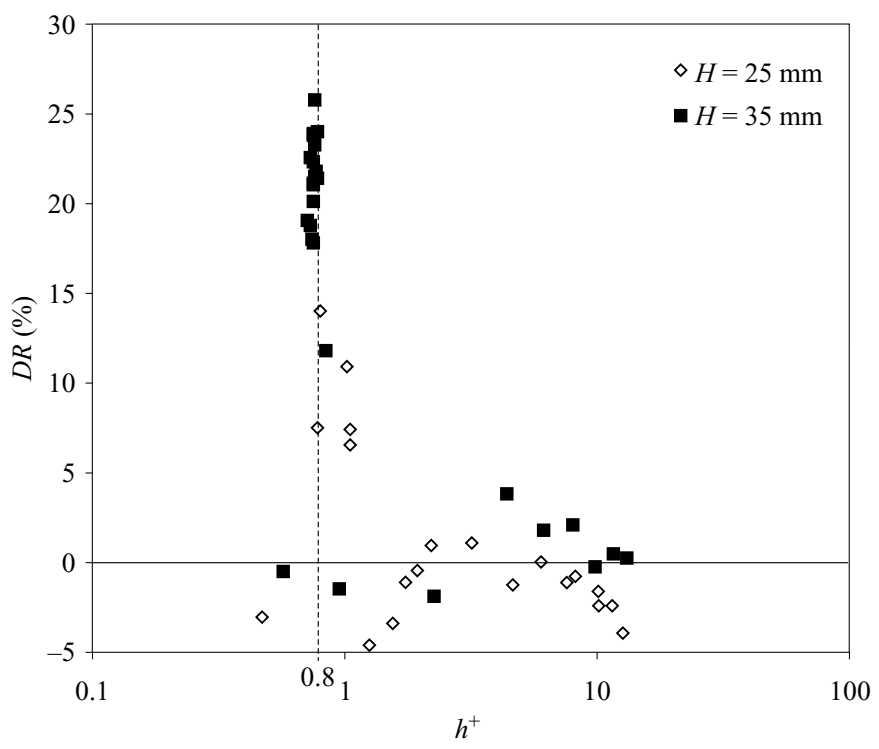

FIGURE 11. Drag reduction plotted versus dimensionless groove size.

The drag reduction results for $H=35 \mathrm{~mm}$ are plotted in figure 10 . As for the first set of measurements, a sharp peak exists in the data where high drag reduction was found. For this larger channel height, $D R$ up to $25 \%$ was measured repeatedly. Based on repeatable measurements, it was concluded that significant drag reduction was achieved in the range $R e_{b}=2700-3200$. In dimensionless groove size, this corresponds to the finding of the first set of experiments: high drag reduction is found in a narrow region for groove sizes below $h^{+}=1$.

Figure 11 summarizes the two sets of experiments, plotted versus the dimensionless size of the grooves. For both the region where high drag reduction was measured is located around $h^{+} \approx 0.8$. It is interesting that high values of drag reduction were found only for groove sizes which correspond to about one viscous length scale or approximately half of the Kolmogorov scale. This might seem surprising since wall roughness below the size of the viscous sublayer $\delta\left(\delta^{+} \simeq 5\right)$ is usually considered to be 
hydraulically smooth and therefore without influence on the resulting pressure drop in channel or pipe flows.

\section{Conclusions}

Based on previous analytical and numerical investigations a surface modification with grooves inserted in the flow direction is proposed. This surface structure is shown to realize the high values of drag reduction reported for numerical simulations with forced boundary conditions (Frohnapfel et al., 2007). The performance of the grooved surface in drag reduction was tested in an experimental facility in which the pressure drop over a channel test section was measured. The first part of the test section had a grooved surface and the second part had smooth channel walls. The comparison of the pressure drops for each part was used to calculate the drag reduction. In experiments at low Reynolds numbers, drag reduction up to $25 \%$ was measured, which is significantly higher than any values reported so far for flow control with surface modifications.

This work is sponsored by the Deutsche Forschungsgemeinschaft under grant number Jo240/5-1. The authors gratefully acknowledge this support. We would like to thank Dipl.-Ing. Hermann Lienhart and Mr Claus Bakeberg for their valuable help in the laboratory and the careful construction of the experimental set-up.

\section{REFERENCES}

Bewley, T. R., Moin, P. \& Temam, R. 2001 DNS-based predictive control of turbulence: an optimal benchmark for feedback algorithms. J. Fluid Mech. 447, 179-225.

Chol, H., Morn, P. \& Kiм, J. 1993 Direct numerical simulation of turbulent flow over riblets. J. Fluid Mech. 225, 503-539.

Durst, F., Fischer, M., Jovanović, J. \& Kikura, H. 1998 Methods to set up and investigate low Reynolds number, fully developed turbulent plane channel flows. Trans. ASME: J. Fluids Engng 120, 496-503.

FISCHER, M. 1999 Turbulent wall-bounded flows at low Reynolds numbers (in German). PhD thesis, University of Erlangen-Nuremberg.

FroHNAPFEL, B. 2007 Flow control of near-wall turbulence. PhD thesis, University of ErlangenNuremberg, Shaker Verlag, Aachen.

Frohnapfel, B., Lammers, P., Jovanović, J. \& Durst, F. 2007 Interpretation of the mechanism associated with turbulent drag reduction in terms of anisotropy invariants. J. Fluid Mech. 577, 457-466.

Jovanović, J. \& Hillerbrand, R. 2005 On peculiar property of the velocity fluctuations in wallbounded flows. J. Thermal Sci. 9, 3-12.

Jovanović, J., Pashtrapanska, M., Frohnapfel, B., Durst, F., Koskinen, J. \& Koskinen, K. 2006 On the mechanism responsible for turbulent drag reduction by dilute addition of high polymers: theory, experiments, simulations, and predictions. Trans ASME: J. Fluids Engng 128, 118-130.

Lee, K. H., Cortelezzi, L., Kim, J. \& Speyer, J. 2001 Application of reduced-order controller to turbulent flows for drag reduction. Phys. Fluids 13, 1321-1330.

Lumley, J. L. \& Newman, G. 1977 The return to isotropy of homogeneous turbulence. J. Fluid Mech. 82, 161-178.

WaLsh, M. J. 1980 Drag reduction of V-groove and transverse curvature riblets. Viscous Drag Reduction (ed. G. R. Hough). Prog. Astronaut. Aeronaut. 72, 168-184. 